\title{
Late Capsular Hematoma after Prosthesis Removal Following Aesthetic Breast Augmentation: A Case
} Report

\author{
Si Hyun Park ${ }^{1}$, Eun Soo Park ${ }^{2}$, \\ Sang Gue Kang ${ }^{1}$ \\ ${ }^{1}$ Department of Plastic and \\ Reconstructive Surgery, Soonchunhyang \\ Seoul Hospital, Soonchunhyang \\ University College of Medicine, Seoul; \\ ${ }^{2}$ Department of Plastic and \\ Reconstructive Surgery, Soonchunhyang \\ Bucheon Hospital, Soonchunhyang \\ University College of Medicine, Bucheon, \\ Korea
}

This work was supported by the Soonchunhyang University Research Fund.

No potential conflict of interest relevant to this article was reported.

\begin{abstract}
Late capsular hematoma formation after augmentation mammoplasty with an implant is a very rare complication. Some mechanisms explaining late capsular hematoma formation have been reported; it is thought to be associated with capsular contracture, textured implants, and the use of corticosteroid and anticoagulant agents. However, no reports of late capsular hematoma formation after prosthesis removal have been published. Herein, we report a case of late capsular hematoma formation after the removal of a prosthesis 10 years previously, following augmentation mammoplasty.
\end{abstract}

Keywords Anticoagulants, Breast implants, Hematoma, Postoperative complications

\section{INTRODUCTION}

Augmentation mammoplasty with prosthesis implantation may cause several early and late complications. Among the early complications, hematoma formation is common, with an incidence ranging from $2 \%$ to $10.3 \%$; it usually takes place approximately 3 days postoperatively [1]. Late hematoma formation is very rare, and has only been reported in a few cases. Its incidence and etiology are still unclear. The interval from surgery to the onset of late hematoma has been reported to range from 6 months to 12 years [1-4]. Herein, we report the first case of late capsular hematoma

Received: Feb 13, 2017 Revised: Apr 9, 2017 Accepted: Apr 10, 2017 Correspondence: Sang Gue Kang Department of Plastic and Reconstructive Surgery, Soonchunhyang University Seoul Hospital, Soonchunhyang University College of Medicine, 59 Daesagwan-ro, Yongsan-gu, Seoul 04401, Korea. E-mail: sgkang@schmc.ac.kr

Copyright $\odot 2017$ The Korean Society for Aesthetic Plastic Surgery.

This is an Open Access article distributed under the terms of the Creative Commons Attribution Non-Commercial License (http://creativecommons.org/licenses/by-nc/4.0/) which permits unrestricted non-commercial use, distribution, and reproduction in any medium, provided the original work is properly cited. $\quad w w w . e-a a p s . o r g$ formation because of newly started anticoagulation 10 years after prosthesis removal, following augmentation mammoplasty that was performed 20 years ago. After the patient underwent magnetic resonance imaging (MRI), we carried out total excision of the capsule. In this report, the clinical, radiological, gross examination, and histological features of this case are presented.

\section{CASE REPORT}

A 70-year-old woman visited us complaining of discomfort due to a 2-month history of a palpable mass on her left breast. She was worried about the palpable mass being cancerous. The symptoms started spontaneously, and no traumatic event had taken place. The patient had undergone augmentation mammoplasty with implants 20 years previously, and she had the implants removed because of capsular contracture 10 years ago. Moreover, she had a 15year history of diabetes mellitus and hyperlipidemia, which were well controlled by oral medication. Her clinical history revealed that she was taking $15 \mathrm{mg}$ of rivaroxaban (a direct inhibitor of the coagulation factor $\mathrm{Xa}$ ) twice a day because of deep vein thrombosis. She started taking the medicine after being diagnosed with deep 

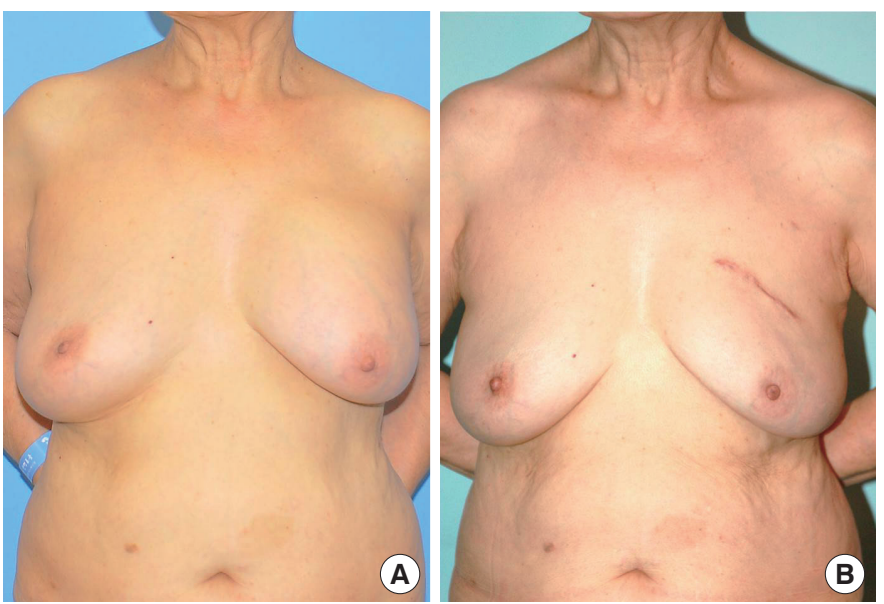

Fig. 1. Preoperative and postoperative photographs. The preoperative photograph shows a bulged upper pole in the left breast caused by a mass measuring $10 \times 5 \mathrm{~cm}$ (A). Postoperative photograph taken 1 month after the operation (B).
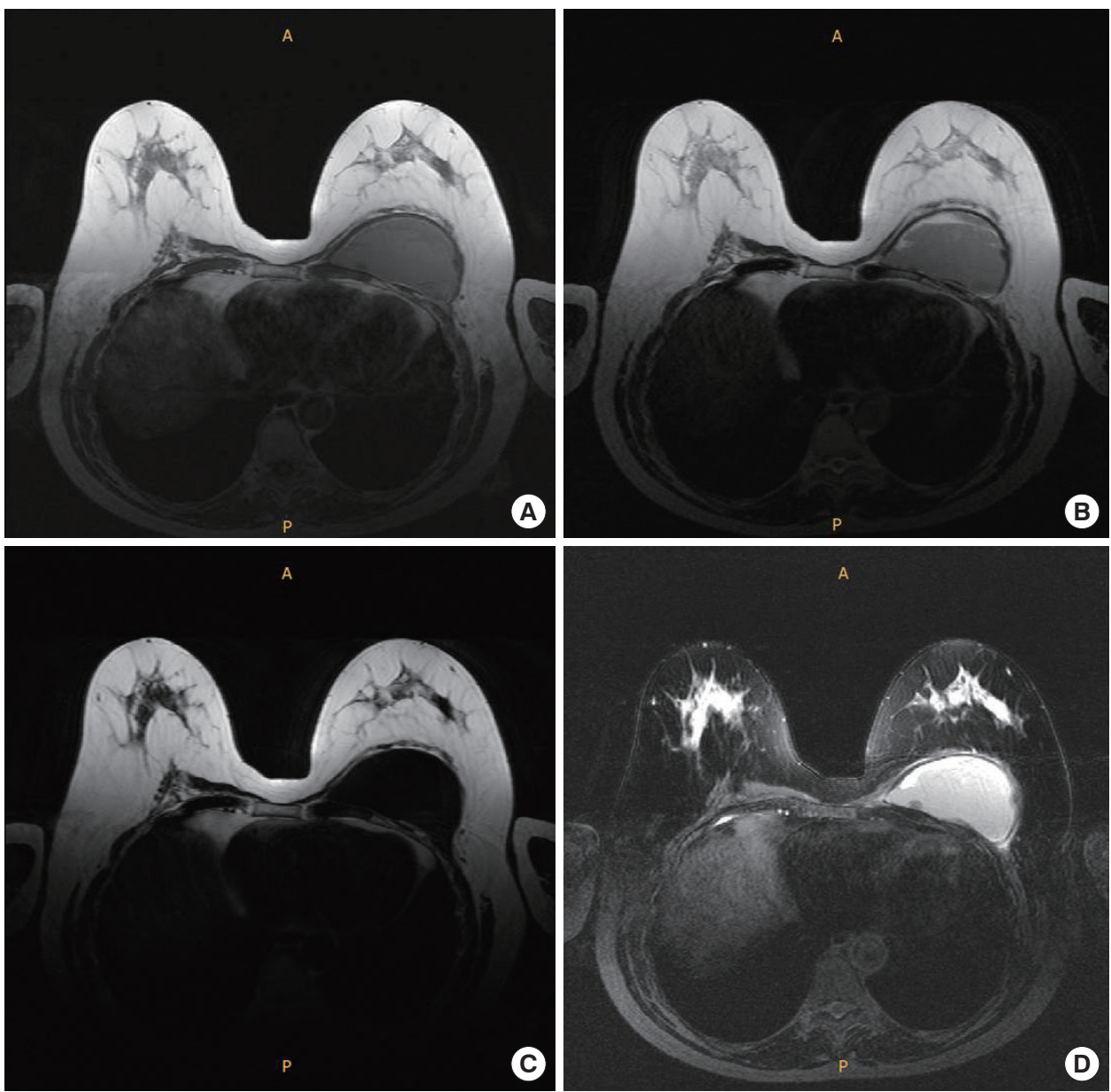

Fig. 2. Magnetic resonance imaging. A capsulated lesion measuring $8.8 \times 4.5 \mathrm{~cm}$, between the pectoralis muscle and the anterior chest wall; the inside of the lesion showed high signal intensity on a T1-weighted image (WI) (A) and a T2-WI (B). The presence of low signal intensity inside the lesion in T2-WI (water-suppressed) imaging (C) indicates that most of the lesion was filled with fluid. Several areas with low signal intensity within the lesion in T2-WI (fat-suppressed) imaging (D) indicate the presence of focal fat components. 

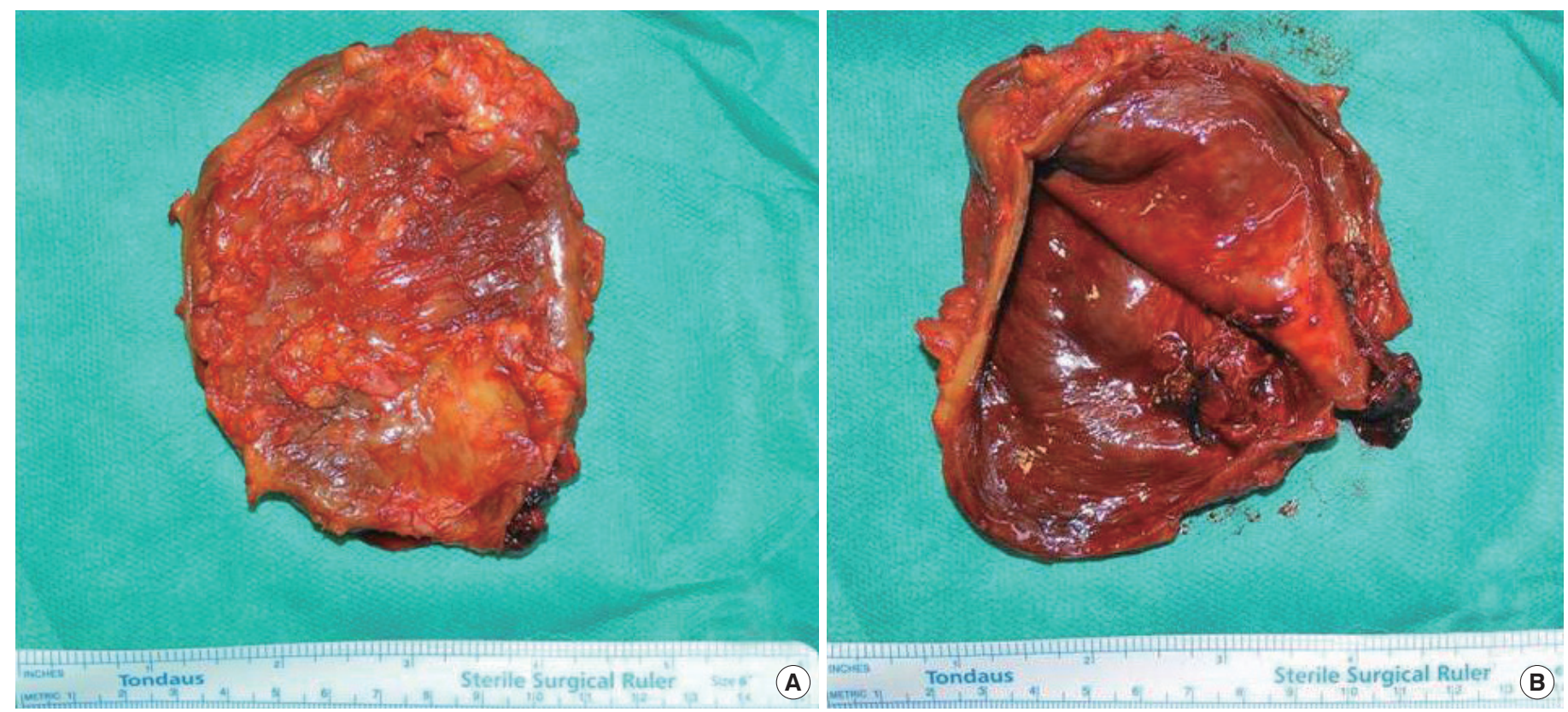

Fig. 4. Gross findings of the capsular hematoma. The cyst consisted of reddish-brown soft tissue, weighing $68 \mathrm{~g}$ and measuring $10 \times 8 \times 1.2 \mathrm{~cm}$ (A). The cyst was opened with a blade, and multiple fragments of brown soft tissue were found inside (B).

vein thrombosis 4 months before presenting to our clinic. The palpable mass measured $10 \times 5 \mathrm{~cm}$ in size and was ovoid, nontender, soft, and movable. No bruise, discoloration, erythema, or signs of inflammation on the covering skin were observed (Fig. 1). In order to evaluate the mass, we performed breast MRI with contrast enhancement. No abnormally enhancing mass was observed in the breast parenchyma, but a capsulated lesion measuring $8.8 \times 4.5 \mathrm{~cm}$ was seen between the pectoralis muscle and the anterior chest wall. The inside of the lesion showed high signal intensity on a T1-weighted image (WI) and a T2-WI. The radiologist concluded that the lesion was a the result of a foreign body reaction, and was composed of water and a small amount of fat between the pectoralis muscle and the left chest wall (Fig. 2). However, no lesion was observed on a previous chest computed tomography (CT) scan of the patient, which had been carried out 4 months previously when the patient was diagnosed with a pulmonary embolism. The observed mass was completely excised under general anesthesia. Intraoperative findings revealed a cyst between the pectoralis major and the anterior chest wall. No active bleeding or enlarged vessels were identified (Fig. 3). The gross findings of the cyst consisted of reddishbrown soft tissue; it weighed $68 \mathrm{~g}$ and measured $10 \times 8 \times 1.2 \mathrm{~cm}$. We opened the cyst with a blade and found multiple fragments of brown soft tissue inside (Fig. 4). The subpectoral space was irrigated and sutured with drain insertion. The operation was finished after cutaneous repair and aseptic compressive dressing. Microscopic findings revealed a cyst lined by fibrous tissue with a foreign body reaction, chronic nonspecific inflammation, and fibrin deposition consistent with a reaction to the implant and subsequent hemorrhage. The patient underwent daily dressings until the removal of the drain and all the stitches 7 days postoperatively. Although she did not discontinue anticoagulant therapy at any time, no recurrence was observed at a 2-month follow-up examination (Fig. 1).

\section{DISCUSSION}

Several mechanisms of late capsular hematoma formation have been suggested. Labadie and Glover [5] described the formation of a late expanding hematoma caused by a chronic inflammatory response and increased vascular permeability. Wang et al. [3] found that in several cases, textured implants stimulated an aggressive foreign body inflammatory reaction, resulting in prolonged high vascularity. Georgiade et al. [1] found an actively bleeding medium-sized vessel in their re-exploration surgery. They suggested that the corticosteroid injected into the implant caused vessel erosion directly. Marques et al. [6] suggested that a capsular microfracture was associated with the rigidity of the capsule, which may have prevented constriction of the injured vessels, leading to bleeding. In our case, the start of anticoagulant therapy 4 months ago was noteworthy. Late hematomas have been reported secondary to anticoagulant therapy with warfarin in spite of a well-controlled international normalized ratio [7].

The treatment of capsular contracture after breast augmentation is variable. Capsulectomy is the most effective method for removing a capsule, but it poses the risk of distortion of the overlying skin, compromised cutaneous blood supply, bleeding, muscle injury, 
and/or pneumothorax [8]. If a patient simply wants the implant to be removed without reinsertion, the treatment of choice is the removal of at least a portion of the capsule with simultaneous mastopexy for the reduction of redundant skin and soft tissue [9]. If there is capsular calcification or implant rupture, capsulectomy is recommended [8]. In our case, we were not able to obtain the previous operative findings of the patient, including the severity of capsular contracture, capsular calcification, and details regarding the rupture of the inserted implant, because the patient underwent both breast augmentation and implant removal surgery a very long time ago in an aesthetic clinic that is now out of business. Judging from the fact that there was no scar tissue on the skin or inside the opened cyst, we suspect that only implant removal was performed, without other procedures such as scoring and/or mastopexy.

With respect to the pathophysiology of our case, we believe that the vessels of capsule did not shrink sufficiently because of the rigidity of the capsule and the fact that the capsule was not removed during the operation for prosthesis removal 10 years ago; the vessel started to bleed again because of the newly taken anticoagulant. No similar cases of late capsular hematoma at a long interval after implant removal have been previously reported.

The most common symptom of patients with late capsular hematoma is painful swelling of the breast. Skin discoloration and sinus tract discharge are less common. Symptoms of infection are rare. Moreover, if the mass is large, an asymmetry between the breasts can be seen [2]. Our patient presented with a painless palpable mass, and she was worried about the palpable mass being cancerous. Several reports documenting an association between the presence of a prosthesis and anaplastic large-cell lymphoma have been published [10]. However, other studies have reported that women who underwent breast augmentation with silicone implants had a relatively low incidence of breast cancer [11]. Therefore, physicians must consider the possibility of cancer even when the palpable mass is clinically unlikely to be a malignancy.

CT, MRI, and ultrasonography are possible diagnostic tools for such scenarios. Ultrasonography has the major advantage of being able to differentiate among seroma, blood, and a clotted hematoma [12]. MRI is regarded as the best diagnostic tool because of its ability to assess the integrity of the implant [13]. In this case, the patient underwent MRI, and we were able to evaluate the quality of the intracapsular fluid using water-suppressed and fat-suppressed images.

In our case, the capsular hematoma was completely evacuated, as the patient wanted the mass to be removed because of her anxiety about the possibility of cancer and a recurrence of the cyst. Some authors have reported carrying out a percutaneous drain guided by ultrasonography; this is a safe and inexpensive procedure when performed by an experienced physician, and has a minimal risk of implant puncture [12]. Hsiao et al. [4] reported 2 cases in which an endoscope was used to inspect the capsule after hematoma evacu- ation and implant removal through a small incision.

In conclusion, when physicians evaluate patients with a palpable breast mass, thorough history-taking and a physical examination must be carried out, keeping in mind the possibility of cancer. Thereafter, MRI can be useful for the differential diagnosis. When late capsular hematoma is suspected on the basis of radiological findings, complete excision of the cyst is recommended to eliminate the possibility of relapse or capsule enlargement. Furthermore, in order to reduce the possibility of capsular hematoma formation, if patients want the implant to be removed without reinsertion, it is necessary to remove at least a portion of the capsule via partial capsulectomy or total capsulectomy.

\section{PATIENT CONSENT}

Patients provided written consent for the use of their images.

\section{REFERENCES}

1. Georgiade NG, Serafin D, Barwick W. Late development of hematoma around a breast implant, necessitating removal. Plast Reconstr Surg 1979;64:708-10.

2. Brickman M, Parsa NN, Parsa FD. Late hematoma after breast implantation. Aesthetic Plast Surg 2004;28:80-2.

3. Wang BH, Chang BW, Sargeant R, et al. Late capsular hematoma after breast reconstruction with polyurethane-covered implants. Plast Reconstr Surg 1998;102:450-2.

4. Hsiao HT, Tung KY, Lin CS. Late hematoma after aesthetic breast augmentation with saline-filled, textured silicone prosthesis. Aesthetic Plast Surg 2002;26:368-71.

5. Labadie EL, Glover D. Physiopathogenesis of subdural hematomas. Part 1: Histological and biochemical comparisons of subcutaneous hematoma in rats with subdural hematoma in man. J Neurosurg 1976; 45:382-92.

6. Marques AF, Brenda E, Saldiva PH, et al. Capsular hematoma as a late complication in breast reconstruction with silicone gel prostheses. Plast Reconstr Surg 1992;89:543-5.

7. Willens HJ, Wald H, Kessler KM. Late intracapsular hemorrhage in an anticoagulated patient with a breast implant. Chest 1996;110:304-5.

8. Young VL. Guidelines and indications for breast implant capsulectomy. Plast Reconstr Surg 1998;102:884-91.

9. Netscher DT, Sharma S, Thornby J, et al. Aesthetic outcome of breast implant removal in 85 consecutive patients. Plast Reconstr Surg 1997; 100:206-19.

10. Thompson PA, Prince HM. Breast implant-associated anaplastic large cell lymphoma: a systematic review of the literature and mini-meta analysis. Curr Hematol Malig Rep 2013;8:196-210.

11. Berkel H, Birdsell DC, Jenkins H. Breast augmentation: a risk factor for breast cancer? N Engl J Med 1992;326:1649-53.

12. Shafir R, Heyman Z, Tsur H, et al. Ultrasound scanning as an aid in 
the diagnosis and treatment of periprosthetic hematoma after breast surgery. Plast Reconstr Surg 1983;71:858-60.

13. Alanen A, Nummi P. Effect of motion on the sonographic and mag- netic resonance patterns of ageing blood. Acta Radiol Diagn (Stockh) 1986;27:455-8. 